\title{
Non-competitive resource exploitation within-mosquito shapes evolution of malaria virulence
}

G. Costa ${ }^{1}$, M. Gildenhard ${ }^{1 \#}$, M. Eldering ${ }^{1,2 \#}$, R.L. Lindquist ${ }^{3}$, A.E. Hauser ${ }^{3,4}$, R. Sauerwein $^{2}$, C. Goosmann ${ }^{1}$, V. Brinkmann ${ }^{1}$ and E.A. Levashina ${ }^{1 *}$

\section{Affiliations:}

${ }^{1}$ Max Planck Institute for Infection Biology, Berlin, Germany

${ }^{2}$ Radboud University Medical Center, Nijmegen, The Netherlands

${ }^{3}$ German Rheumatism Research Centre (DRFZ), Berlin, Germany

${ }^{4}$ Charité-Universitätsmedizin Berlin, Germany

\# These authors contributed equally to this work

* Corresponding author: levashina@mpiib-berlin.mpg.de 


\section{Abstract}

3 Malaria is a fatal human parasitic disease transmitted by a mosquito vector. The 4 evolution of within-host malaria virulence has been the focus of many empirical and 5 theoretical studies. However, the vector's contribution to virulence evolution is not well 6 understood. Here we explored how within-vector resource exploitation impacts 7 evolutionary trajectories of within-host Plasmodium virulence. We developed a nested 8 model of within-vector dynamics and malaria epidemiology, which predicted that non9 competitive resource exploitation within-vector restricts within-host parasite virulence. To 10 validate our model, we experimentally manipulated mosquito lipid trafficking and gauged 11 within-vector parasite development, within-host infectivity and virulence. We found that 12 mosquito-derived lipids determine within-host parasite virulence by shaping development 13 and metabolic activity of transmissible sporozoites. Our findings uncover the role of 14 within-vector environment in regulating within-host Plasmodium virulence and identify 15 Plasmodium metabolic traits that may contribute to the evolution of malaria virulence. 


\section{INTRODUCTION}

18 Malaria is caused by the vector-borne protozoan parasite Plasmodium falciparum and

19 kills 429,000 people annually, predominantly in sub-Saharan Africa ${ }^{1}$. The unacceptably

20 high malaria mortality is tightly linked to within-host parasite virulence (here defined as

21 capacity to cause harm to the human host). Therefore, predicting the evolution of

22 Plasmodium virulence has important epidemiological, socio-medical and evolutionary

23 implications for designing malaria control strategies. The life cycle of malaria parasites is

24 split between the host and the mosquito vector. Theoretical and empirical studies

25 focused on regulation of within-host malaria virulence, including contributions of parasite

26 genetic factors and host immune responses, and on the link between virulence and host-

27 to-vector transmission ${ }^{2-4}$. The latter reports showed that genetically-encoded within-host

28 parasite virulence increases the production of infective to vector forms and, thereby,

29 parasite transmission to the vector ${ }^{5}$. Females of anopheline mosquitoes feed on blood to

30 initiate ovary development. Therefore, only females ingest Plasmodium and contribute to

31 malaria transmission. During a blood meal, blood-borne sexual forms of parasites fuse to

32 form motile zygotes that within two days after infection traverse the midgut epithelium,

33 reach the basal side of the midgut to round-up into oocysts. In the next 10-15 days, each

34 oocyst undergoes sporogony, a process that generates thousands sporozoites, the

35 parasite stage infective to humans. Sporozoites egress from the oocysts and migrate to

36 the mosquito salivary glands to reach full maturity and infectivity ${ }^{6}$. Nutrients taken up by

37 female mosquitoes during a blood meal are essential for vector reproduction and for

38 massive Plasmodium proliferation at the oocyst stage ${ }^{7,8}$. Although many studies

39 examined associations between Plasmodium virulence and vector fitness, only a few

40 reported significant changes in longevity and fecundity of Plasmodium-infected

41 mosquitoes, thus precluding generalized conclusions ${ }^{9-13}$. Whether within-vector resource

42 exploitation by parasites modulates within-host malaria virulence has never been

43 explored.

44 Here we applied theoretical and experimental approaches to examine the link between

45 parasite within-vector resource exploitation and virulence towards the mammalian host.

46 We modeled alternative scenarios of vector-parasite symbiosis and investigated their

47 consequences for parasite transmission dynamics. Our modeling results predicted that

48 vector-parasite relationship of resource acquisition shapes the evolution of within-host

49 virulence. A parasitic relationship, where the parasite exploited vector mechanisms to 
50 scavenge resources, restricted parasite virulence, whereas virulence runaway was

51 observed in a competitive relationship. To test these predictions, we experimentally

52 manipulated lipid trafficking in genetically identical mosquitoes and exploited a rodent

53 malaria model to gauge the impact of these manipulations on within-host virulence of

54 genetically identical parasites. We found that mosquito-derived lipids determine within-

55 host Plasmodium virulence by shaping sporogony and metabolic activity of sporozoites.

56 By showing that evolution of malaria virulence is metabolically regulated by the within-

57 vector nutritional environment, our findings have important implications for vector control

58 strategies based on biological competitors or on manipulation of mosquito reproduction.

\section{RESULTS}

\section{Within-vector environment shapes evolution of within-host malaria virulence}

62 To test for the evolutionary consequences of a trade-off between mosquito reproduction, 63 parasite reproduction and virulence, we used a modeling approach by considering a 64 within-vector dynamics model in an epidemiological framework (Materials and Methods).

65 We modeled within-vector resource competition between mosquito reproduction (egg 66 production) and parasite reproduction (sporozoite load) as a competitive Lotka-Volterra 67 equation ${ }^{14,15}$. This model describes egg production, parasite production and the 68 competition strength between vector and parasite $\alpha_{E P}$ and $\alpha_{P E}$ (their ability to inhibit 69 each other's growth). Mechanistically, the parasite's competition coefficient represents 70 its within-vector resource scavenging and, thereby, its harm towards vector reproduction.

71 We next combined this within-vector model with an epidemiological model for vector72 borne disease, which describes human-to-vector and vector-to-human parasite 73 transmission. Pathogen evolution can be investigated with epidemiological models, 74 assuming that selection maximizes the parasite's ability to spread from one host to the 75 next expressed as the basic reproductive ratio $R_{0}$. For vector-borne diseases, $R_{0}$ is the 76 product of the vector-to-host and host-to-vector transmission potentials ${ }^{16}$. In line with 77 previous reports ${ }^{17-19}$, we postulate that virulence towards the host is influenced by within78 vector resource acquisition. We specifically hypothesize that within-vector resource 79 exploitation influences virulence by impacting parasite load and infectivity to the host 80 (Figure 1A). 
81 Combining within-host Lotka-Volterra dynamics with epidemiological dynamics allows us

82 to investigate evolution of $\mathrm{R}_{0}$ under varying parasite and vector competition coefficients.

83 When vector and parasite use independent mechanisms to scavenge resources

84 (competitive relationship), both competition coefficients have a positive sign ( $\alpha_{E P}>$

$850 ; \alpha_{P E}>0$ ), reflecting a reciprocally inhibitory competitive relationship (Figure $1 \mathrm{~B}$ ). We

86 found that competitive environment selects for higher parasite's competition coefficient

87 (Figure 1C), eventually leading to vector sterilization and to high levels of within-host

88 virulence (Figure 1D). This can be explained by the fact that both vector-to-human and

89 human-to-vector transmission rates benefit from increasing the parasite's

90 competitiveness. When parasites mechanistically depend on the vector's investment in

91 reproduction (parasitic relationship), the vector's competition coefficient turns negative

$92\left(\alpha_{E P}>0 ; \alpha_{P E}<0\right)$ (Figure 1C). We found that in contrast to the competitive scenario, the

93 parasitic relationship leads to opposing evolutionary trajectories between the two

94 transmission events. Indeed, higher $\alpha_{E P}$ decreases parasite's density and vector-to-

95 human transmission but increases human-to-vector transmission (Figure 1E). Such a

96 trade-off between transmission events would lead to the emergence of an evolutionarily

97 stable malaria virulence (Figure $1 \mathrm{C}$ and $\mathrm{D}$ ).

98 Taken together, our theoretical analyses predicted that the shape of vector-parasite

99 relationship defines the evolutionary trajectories of within-host virulence.

101 Mosquito lipid environment regulates Plasmodium virulence in the next 102 mammalian host

103 To formalize our theoretical assumption that within-host virulence is defined by within104 vector resource acquisition, we examined changes in Plasmodium virulence after 105 restricting within-vector lipid resources. In these experiments, we used RNAi in adult 106 females to deplete the major mosquito lipid transporter lipophorin (LP) without affecting 107 mosquito diet or development ${ }^{20-22}$. As expected, Lp depletion prevented lipid transport 108 between mosquito tissues, inhibited ovary and parasite development (Figure S1). We 109 next exposed naïve mice to the bites of $P$. berghei-infected control or Lp-depleted 110 mosquitoes. We found that $\mathrm{Lp}$ depletion drastically attenuated parasite infectivity and 111 virulence. Indeed, only $20 \%$ of mice bitten by Lp-depleted mosquitoes became infected 
112 and only $10 \%$ developed severe neurological symptoms of experimental cerebral

113 malaria (ECM) compared to $90 \%$ in controls (Figure $2 \mathrm{~A}-\mathrm{C}$ ). As the observed phenotypes

114 could result from the differences in numbers of inoculated parasites, we subcutaneously

115 injected naïve mice with equal quantities of sporozoites isolated from control and Lp-

116 depleted mosquitoes. Again, fewer than $40 \%$ of mice injected with lipid-deprived

117 sporozoites became infected and only $20 \%$ of mice developed ECM, as compared to

$118100 \%$ in controls (Figure 2D-F). These results confirm the major assumption of our

119 model and show that the within-vector lipid environment impacts within-host parasite 120 virulence.

122 Plasmodium parasitizes mosquito lipids for productive sporogony and formation 123 of infective sporozoites

124 Our theoretical study predicted that the type of symbiotic relationship (competitive or 125 parasitic) defines the evolutionary trajectory of parasite virulence. As restricting 126 trafficking of lipid resources impacted virulence, we examined the parasitic scenario, 127 where Plasmodium exploits mosquito Lp for lipid delivery. We microscopically gauged 128 lipid accumulation and parasite growth using Nile Red lipid staining, and observed 129 accumulation of neutral lipids in the peripheral cytoplasm and vesicles of mature oocysts 130 in control mosquitoes. In contrast, levels of Nile Red staining were significantly lower in 131 the oocysts of Lp-depleted females (Figure $3 A$ and $B$ ). Moreover, the 132 immunofluorescence analysis with anti-Lp antibodies in controls showed an increase in 133 the rate of Lp-positive oocysts from $25 \%$ to $72 \%$ at 7 and 13 days post infection (dpi), 134 respectively (Figure S2). These results support the parasitic scenario, in which 135 Plasmodium exploits $\mathrm{Lp}$ for lipid delivery to its proliferating stages. Depletion of $\mathrm{Lp}$ 136 inhibited oocyst sporulation, caused abnormal cytoplasmic vacuolizations (Figure 3C 137 and Figure S3), and significantly reduced the size of mature oocysts (Figure 3D). In line 138 with these results, perturbation of lipid trafficking decreased the density of transmissible 139 sporozoites (Figure 3E). Similar results were obtained with the human malaria parasite $140 P$. falciparum (Figure 3D and E), suggesting that within-vector parasitic relationships are 141 conserved between human and rodent Plasmodium species.

142 As lipid restriction attenuated within-host virulence, we set out to identify Plasmodium 143 traits affected by Lp depletion. In these experiments, we compared morphology, vitality 
144 and gene expression of the sporozoites produced by control and Lp-depleted

145 mosquitoes. We found that lipid-deprived sporozoites were alive and properly expressed

146 major maturation markers (Figure 3F and Figure S4). As lipids are crucial for metabolic

147 activity, we measured mitochondrial membrane potential using TMRE dye, which

148 accumulates in the membrane matrix of active mitochondria ${ }^{23}$. Lipid-deprived

149 sporozoites showed a significant decrease in mitochondrial membrane potential

150 compared to controls (Figure 3G and Figure S4), suggesting that mosquito lipids shape

151 metabolic activity of Plasmodium transmissible forms. Moreover, TMRE intensities in

152 control sporozoites were broadly distributed over a range of 2.5 logs, as opposed to a

153 sharp peak of the cytoplasmic GFP signal (Figure 3F and G). These results suggest that

154 genetically identical sporozoites display high variability in mitochondrial activity, which

155 may contribute to the plasticity of within-host virulence. Taken together, our data provide

156 experimental evidence that Plasmodium exploits $L p$ to scavenge mosquito lipid

157 resources for its within-vector proliferation in a non-competitive parasitic manner, and

158 identify mitochondrial membrane potential as a virulence-associated trait.

160 Time-shift in Plasmodium sporulation circumvents competition for mosquito 161 resources

162 What mechanism allows the parasites to avoid competition for the vector's resource 163 investment in reproduction? To answer this question, we gauged the timing of lipid 164 consumption by the mosquito ovaries and of parasite proliferation. We observed a clear 165 temporal shift between these two processes. While mosquito ovaries accumulated lipids 166 within the first two days after feeding, Plasmodium proliferation began one week post167 infection (Figure 4A). We hypothesized that in order to avoid a direct competition for 168 nutrients, Plasmodium could have evolved to delay its replication until after completion of 169 vector's oogenesis. This time-shift hypothesis is compatible with the non-competitive 170 parasitic scenario where parasites benefit from lipid delivery after completion of 171 oogenesis, as long as sufficient resources are left for parasite development. Therefore, if 172 lipid restriction affected only replicating oocysts, providing lipids at the initiation of oocyst 173 sporogony should bypass one week of lipid starvation and rescue parasite development 174 and virulence. We tested this hypothesis by directly gauging sporozoite infectivity and 175 development into extra-erythrocytic forms (EEFs) in the hepatoma HepG2 cells in vitro. 
176 Cells were exposed to equal numbers of sporozoites isolated from control and Lp-

177 depleted mosquitoes that received (Figure 4Bv-viii) or not (Figure 4Bi-iv) a supplemental

178 blood feeding seven days post infection, at the time when the first effects of lipid

179 starvation on the oocyst's size were observed (Figure $3 C$ and Figure S3). EEF

180 development was examined by fluorescence microscopy. As expected for a single blood

181 feeding, lipid restriction attenuated sporozoite numbers, infectivity and development in

182 vitro, as significantly smaller EEFs were produced by lipid-deprived sporozoites

183 compared to controls (Figure 4Bi-iv and Figure S5). Strikingly, the supplemental feeding

184 restored sporozoite numbers in Lp-depleted mosquitoes, as well as parasite infectivity

185 and EEF development (Figure 4Bv-viii and Figure S5). Our data show that delivering

186 lipids to the oocysts after completion of vector's oogenesis rescues Plasmodium

187 infectivity and virulence. Taken together, these results suggest that a time-shift in

188 Plasmodium sporogony circumvents within-vector competition for reproductive

189 resources.

\section{DISCUSSION}

192 Virulence is one of the most evolutionarily relevant and complex pathogen traits.

193 Understanding virulence mechanisms provides an opportunity to predict and, potentially

194 control the pathogen burden and its evolution. Here we generated theoretical models of

195 the mosquito-parasite relationship, experimentally evaluated them, and found that

196 Plasmodium hijacks the main mosquito lipid transporter for lipid delivery to the actively

197 proliferating oocysts after completion of the vector reproductive cycle. Such time-shift in

198 oocyst maturation benefits the parasitic relationship by allowing a timely allocation of

199 resources needed for host reproduction and for parasite proliferation. Temporal

200 regulation plays a critical role in host-parasite interactions and in host immune

201 responses. In mosquitoes, the ookinetes at the basal side of the midgut are the major

202 target of the mosquito immune system within the first 24-48 $\mathrm{h}$ after blood meal, whereas

203 even the earliest oocyst stages are spared from the complement attack ${ }^{24}$. Interestingly,

204 sporogony is the longest replicative process in the life cycle of all Plasmodium species

205 (12 to 16 days depending on the species). Given the average mosquito life span of 30

206 days, it roughly corresponds to a half of mosquito life. Prolongation of the within-vector

207 sejour is risky for the parasite, which should be selected to maximize its chances to 
208 infect the next host as fast as possible. Therefore, the beneficial effects of lengthy 209 sporogony must be under a strong selective pressure. We predict that shifting the timing 210 of resource allocation should have dramatic consequences for parasite development, 211 transmission and virulence.

212 Our observations suggest that limited lipid resources during sporulation induce an 213 autophagy-like cell death and select for the most competitive parasites that effectively 214 scavenge nutrients from the mosquito. Autophagy, often characterized by cytoplasmic 215 vacuolization, has been observed in a large proportion of Plasmodium liver stages ${ }^{25}$, and 216 our results extend these observations to the extracellular oocysts. Nutrient restriction in 217 natural conditions could be induced by oocyst crowding ${ }^{19}$; however in our experiments 218 the numbers of pre-sporogonic oocysts between the experimental groups were $\operatorname{similar}^{21}$. 219 Interestingly, prolonged periods of nutrient deprivation cause cell autophagy and induce 220 mitochondrial dysfunction in mouse embryonic fibroblasts ${ }^{26}$. Similarly, we found that lipid 221 restriction blunted sporozoite's mitochondrial membrane potential. Mitochondria are 222 essential for mosquito stages of the Plasmodium life cycle, as mutations in mitochondrial 223 genes arrest growth and sporulation of oocysts ${ }^{27-30}$. Moreover, inhibition of mitochondrial 224 potential by the antimalarial drug atovaquone inhibits Plasmodium sporogonic 225 development and reduces the number and size of developing EEFs in vitro ${ }^{31-33}$. Together 226 with these findings, our results identify mitochondria as crucial regulators of within-vector 227 Plasmodium proliferation, transmission and within-host virulence. Although drug 228 pressure on the mitochondria frequently selects for resistance, most of the identified 229 mutations are loss-of-function, that disrupt within-vector parasite development and 230 disable transmission ${ }^{30}$. Therefore, Plasmodium mitochondria may represent an 231 evolution-proof drug target ${ }^{34}$, as long as no gain-of-function mutations emerge that may 232 increase mitochondrial activity and, thereby, parasite virulence.

233 At the ecological level, our data show that the plasticity of parasite virulence is 234 metabolically regulated. We found that even in normal conditions, sporozoites display 235 broad variability in their mitochondrial activity. This implies that mosquito life-history traits 236 can directly influence parasite virulence. Variable trophic environments of larval breeding 237 sites $^{35}$ and of blood meals should translate inter-individual mosquito nutritional 238 differences into variability in Plasmodium virulence. Indeed, multiple reports have 239 implicated larval diet and adult blood meals in determining parasite loads within a 240 mosquito ${ }^{18,36-40}$, but this has not been linked to within-vector modulation of parasite 
241 metabolic activity and within-host virulence. Our findings open up a new perspective on

242 mosquito transmission capacity and on evolution of malaria virulence.

243 The evolutionary responses to vector control measures are often non-linear due to 244 complexity of vector-parasite relationships. Competitive within-vector Plasmodium 245 dynamics may result in a virulence runaway, leading to a competitive exclusion between 246 vector and parasite and exacerbating within-host virulence. We show that parasitic non247 competitive exploitation of vector lipid resources restricts evolution of Plasmodium 248 virulence. Importantly, harm towards the vector's reproduction alone does not restrict 249 virulence evolution ${ }^{41}$. The trade-off between within-vector parasite density and within250 host virulence that restrains virulence at intermediate levels, emerges only when 251 parasite resource acquisition depends on vector mechanisms. We provide empirical 252 evidence that within-vector Plasmodium development relies on the major vector lipid 253 transporter and that competition between vector and parasite is prevented by a time-shift 254 between vector oogenesis and parasite sporogony. This beneficial developmental niche 255 should be retained as long as nutrient supply is sufficient. In a low nutrient environment, 256 the relationship may switch towards competition, which would rapidly invert the trajectory 257 of virulence evolution. Further investigation of the effects of other mosquito factors on 258 parasite resource acquisition is necessary to predict potential switches between parasitic 259 and competitive vector-parasite relationships. Such situations could arise from targeting 260 mosquito reproduction factors essential for parasite development by transgenic 261 approaches $^{42}$, or from introduction of such biological competitors as Wolbachia ${ }^{43}$. All 262 together our data highlight the links between metabolism and life-history traits of vector 263 and parasite, that shape evolutionary trajectories of malaria virulence. Further 264 theoretical, experimental, and field studies are needed to determine the effects of natural 265 and artificial perturbations of vector and parasite metabolism on malaria epidemiology. 


\section{MATERIALS AND METHODS}

\section{Mathematical modeling}

\section{Within-vector dynamics}

269 Vector and parasite compete for resources from a single blood meal (non-replenished

270 nutrient source). To investigate how vector-parasite relationship shapes within-host

271 virulence, we first modeled resource competition between vector reproduction and

272 sporozoite development. For this purpose, we use a Lottka-Volterra competition

273 model $^{14,15}$, considering inter- and intraspecific competition between mosquito eggs $(E)$

274 and parasites $(P)$ :

$275 \dot{E}=r_{1} E\left(1-\frac{E+\alpha_{E P} P}{K_{E}}\right)$

$276 \dot{P}=r_{2} P\left(1-\frac{P+\alpha_{P E} E}{K_{P}}\right)$,

277 where $r_{1}$ and $r_{2}$ represent the maximum intrinsic growth rate of eggs and parasites, 278 respectively, and $\alpha_{E P}$ and $\alpha_{P E}$ describe their competition strength. The maximum egg 279 and sporozoite densities are given by the carrying capacities $K_{E}$ and $K_{P}$, which from this 280 point on we assume to be 1.

281 The four equilibrium points are given by

$$
\left(\begin{array}{cc}
E \rightarrow 0 & P \rightarrow 0 \\
E \rightarrow \frac{-1+\alpha_{E P}}{-1+\alpha_{E P} \alpha_{P E}} & P \rightarrow \frac{-1+\alpha_{P E}}{-1+\alpha_{E P} \alpha_{P E}} \\
E \rightarrow 0 & P \rightarrow 1 \\
E \rightarrow 1 & P \rightarrow 0
\end{array}\right)
$$

283 and describe three qualitatively different scenarios: extinction of both species, 284 competitive exclusion, or co-existence. Because both eggs and parasites are found in 285 the mosquito, we hereafter only focus on the non-trivial steady state, i.e., co-existence, 286 which can only occur if both growth rates $\left(r_{1}\right.$ and $\left.r_{2}\right)$ are positive and neither species 287 outcompetes the other, i.e., if $\alpha_{E P}$ and $\alpha_{P E}$ are smaller than 1.

289 For all cases, where $0<\alpha_{E P}<1$; and $0<\alpha_{P E}<1$, sporozoite production $\bar{P}$ decreases 290 with increasing values of $\alpha_{E P}$, and increases with increasing values of $\alpha_{E P}$ for all, where 
$2910<\alpha_{E P}<1 ; 0>\alpha_{P E}<1$ (Figure S6). Instead, vector reproduction $\bar{E}$ should decrease

292 with increasing levels of $\alpha_{E P}$ independent of the sign of $\alpha_{P E}$ (Figure S7).

293 Epidemiological dynamics

294 Following Alizon and van Baalen ${ }^{16}$, Plasmodium epidemiological reproductive ratio $\mathrm{R}_{0}$ is 295 a product of vector-to-host $v \rightarrow h$ and host-to-vector $h \rightarrow v$ transmission rates $\beta$, and of 296 the availability of susceptible hosts $S$. These are constrained by the respective duration 297 of infection defined by parasite induced mortality $\vartheta$, recovery rate $\gamma$, and intrinsic host 298 mortality $\mu$ :

$299 R o=\frac{\beta_{v \rightarrow h}}{\mu_{v}+\vartheta_{v}+\gamma_{v}} S_{v} * \frac{\beta_{h \rightarrow v}}{\mu_{h}+\vartheta_{h}+\gamma_{h}} S_{h}$.

300

$301 \quad$ Nested model

302 To integrate the within-vector dynamics into an epidemiological model of malaria 303 transmission, we first assumed that within-vector sporozoite production is at equilibrium 304 before the time of transmission (i.e., quasi-steady state). Because vector-to-human 305 transmission rate increases with the number of produced sporozoite ${ }^{44}$, we next 306 postulated that vector-to-human transmission rate is a linear function of equilibrium 307 parasite density $\beta_{v \rightarrow h}(\widetilde{P})$ :

$308 \beta_{v \rightarrow h}(\tilde{P})=\theta_{\beta_{v \rightarrow h}} \frac{-1+\alpha_{P E}}{-1+\alpha_{E P} \alpha_{P E}}$,

310 where $\theta_{\beta_{v \rightarrow h}}$ is a constant which describes the maximum transmission rate in relation to 311 parasite density.

312 Host-to-vector transmission depends on the parasites asexual replication and production 313 of sexual forms (gametocytes) ${ }^{5,45}$. Virulence towards the host is caused by the asexual 314 production during the blood stages, which makes host-to-vector transmission a linear 315 function of virulence $\beta_{h \rightarrow v}\left(\vartheta_{h}\right)$. We assume that the capacity to enter the asexual cycle 316 depends on initial parasite load, which links parasite density at the time of vector to 317 human transmission to human to vector transmission:

$318 \beta_{h \rightarrow v}\left(\vartheta_{h}(\tilde{P})\right)=\theta_{\beta_{h \rightarrow v}} \theta_{\vartheta_{h}} \frac{-1+\alpha_{P E}}{-1+\alpha_{E P} \alpha_{P E}}$, 
320 where $\theta_{\vartheta_{h}}$ and $\theta_{\beta_{v \rightarrow h}}$ are the rates for virulence and transmission in relation to parasite

321 loads. However, within-vector resource competition may also qualitatively affect the

322 parasite's capacity to infect the human host ${ }^{17-19}$. Therefore, we assumed that within-host

323 virulence increases with the efficiency of resource exploitation via the parasite's

324 competition coefficient:

$325 \beta_{h \rightarrow v}\left(\vartheta_{h}\left(\alpha_{E P}, \tilde{P}\right)\right)=\theta_{\beta_{h \rightarrow v}} \theta_{\vartheta_{h}} \alpha_{E P} \frac{-1+\alpha_{P E}}{-1+\alpha_{E P} \alpha_{P E}}$

$327 \mathrm{R}_{0}$ under these assumptions is given by equation 8 . It can be used to study the 328 trajectories of virulence evolution in diverse vector-parasite relationships. The parameter 329 values used in this study are provided in Table S1.

\section{Mosquito rearing and parasite Infections}

334 Anopheles gambiae s.l. mosquitoes were used throughout the study. To prevent large 335 losses at early parasite stages (ookinetes) inflicted by the mosquito complement-like 336 system in the absence of $L p^{21,46}$, all infection experiments were performed with immune337 deficient mosquitoes ( $7 \mathrm{~b}$ line $)^{47}$. G3 and $7 \mathrm{~b}$ mosquitoes, were maintained at $29^{\circ} \mathrm{C} 70 \%-$ $33880 \%$ humidity $12 / 12 \mathrm{~h}$ day/night cycle. In $P$. falciparum infections, mosquitoes were fed 339 at $37^{\circ} \mathrm{C}$ for $15 \mathrm{~min}$ on a membrane feeder with NF54 gametocytes cultured with O+ 340 human red blood cells (Haema, Berlin), and, thereafter, kept in a secured S3 laboratory 341 according to the national regulations (Landesamt für Gesundheit und Soziales, project 342 number 411/08). For $P$. berghei experiments, mosquitoes were fed on anesthetized CD1 343 mice infected with the GFP-expressing P. berghei GFP-con 259cl2 clone (ANKA 344 strain $)^{48}$. Shortly after infections, unfed mosquitoes were removed, while fed mosquitoes 345 were maintained at $26^{\circ} \mathrm{C}$ for $11-14$ days $\left(P\right.$. falciparum) or at $20^{\circ} \mathrm{C}$ for $7-21$ days $(P$. 346 berghei), and then used for the midgut and/or the salivary gland dissections.

\section{RNAi silencing}

349 Double stranded RNA (dsRNA) against lipophorin ( $d s L p$ ) was produced as previously 
350 described ${ }^{21}$. For RNAi silencing, 1-2 day-old females were anesthetized with $\mathrm{CO}_{2}$ and 351 injected with $69 \mathrm{nl}$ of $3 \mu \mathrm{g} / \mu \mathrm{l} d s L a c Z$ (control) or $d s L p$ using a Nanoject II Injector 352 (Drummond). Mosquitoes recovered for 3-4 days following injection before infections. 353 Efficiencies of RNAi silencing are summarized in Figure S8.

\section{Immunofluorescence analysis}

356 Mosquito midguts were dissected in PBS, fixed in 4\% formaldehyde and washed in PBS. 357 Tissues were permeabilized with $0.1 \%$ Triton $X-100$ and incubated with a $1: 1$ mix of 358 mouse monoclonal anti-Apol and anti-Apoll antibodies (1/300, clones $2 \mathrm{H} 5$ and 2C6) ${ }^{21}$ 359 overnight at $4^{\circ} \mathrm{C}$ followed by incubation for $40 \mathrm{~min}$ at room temperature with the 360 secondary Cy3-labeled antibodies at 1/1,000 (Molecular Probes), or with $0.1 \mu \mathrm{g} / \mathrm{ml}$ of the 361 Nile Red stain (Sigma Aldrich). Nuclei were stained with DAPI $(1.25 \mu \mathrm{g} / \mathrm{ml}$, Molecular 362 Probes) for 40-60 $\mathrm{min}$ at room temperature. Images were acquired using an 363 AxioObserver Z1 fluorescence microscope equipped with an Apotome module (Zeiss). 364 Oocyst size was measured by hand-designing a circular ROI of randomly selected 365 oocysts (identified by GFP for $P$. berghei or by bright field and DAPI staining for $P$. 366 falciparum) using ZEN 2012 software (Zeiss). Images were then processed by FIJI 367 software (ImageJ $1.47 \mathrm{~m})$.

369 Development of $P$. berghei liver-stages in vitro

370 The salivary glands of $P$. berghei infected mosquitoes were dissected, sporozoites were 371 collected into RPMI medium (Gibco) containing 3\% bovine serum albumin (Sigma 372 Aldrich) and enumerated with a hemocytometer. The $P$. berghei liver stages were 373 cultured in vitro in HepG2 hepatoma cells and analyzed using standard techniques ${ }^{49}$. 374 Briefly, 15,000 - 20,000 HepG2 cells per well (70\% confluence) were plated on 375 transparent-bottom 96-wells plates (Nalgene International) and incubated for $24 \mathrm{~h}$, then 376 seeded with 10,000 sporozoites and co-cultured at $37^{\circ} \mathrm{C}$ for $2 \mathrm{~h}$. Free sporozoites were 377 removed by washing. As a negative control, sporozoites isolated from control 378 mosquitoes were pre-treated with the inhibitor of actin polymerization Cytochalasin D 379 (Sigma Aldrich) for $10 \mathrm{~min}$. At $48 \mathrm{~h}$ post seeding, the cells were fixed with $4 \%$ 380 formaldehyde for $10 \mathrm{~min}$, washed with PBS and blocked with $10 \%$ fetal calf serum in 
381 PBS. Development of the liver-stage parasites was examined using monoclonal mouse 382 anti-GFP antibodies (1/1,000, AbCam) and revealed with the secondary AlexaFluor488 383 conjugated anti-mouse antibody (1/1,000, Molecular Probes). Nuclei were stained with 384 DAPI $(1.25 \mu \mathrm{g} / \mathrm{ml}$, Molecular Probes). Images were recorded directly on the 96-well plate 385 using an AxioObserver Z1 fluorescence microscope equipped with an Apotome module 386 (Zeiss) and analyzed for number and size of liver forms using the Axio-Vision ZEN 2012 387 software (Zeiss).

\section{$P$. berghei mouse infections in vivo}

390 Mice were housed and handled in accordance with the German Animal Protection Law 391 (§8 Tierschutzgesetz) and both institutional (Max Planck Society) and national 392 regulations (Landesamt für Gesundheit und Soziales, registration number H 0027/12).

393 To determine infectivity to mice, the sporozoites collected from the mosquito salivary 394 glands were injected subcutaneously (5,000 sporozoites/mouse) into the tails of 8-10395 week-old C57BL/6 females. Bite-back experiments were performed by feeding the $P$. 396 berghei-infected mosquitoes (18 dpi) on anesthetized naïve C57BL/6 mice (mean 9 397 bites/mouse, range 2-15). Parasitemia was determined by daily Giemsa staining of thin 398 blood smears and FACS analysis of the red blood cells with the GFP-expressing blood 399 stage parasites. Infected mice were monitored every 6-12 $\mathrm{h}$ for the appearance of 400 severe neurological and behavioral symptoms typical of experimental cerebral malaria 401 (ECM) such as hunched body position, grooming alteration, ataxia, paralysis, or 402 convulsions $^{50}$ or by the rapid neurological and behavioral test (RMCBS) ${ }^{51}$. All mice with 403 ECM symptoms or the RMCBS score equal or below 5/20 were sacrificed immediately.

\section{Sporozoite imaging flow cytometry}

406 Salivary gland sporozoites were isolated into RPMI medium (Gibco) 3\% bovine serum 407 albumin (Sigma Aldrich) and kept on ice until staining. Sporozoites were diluted in PBS 408 to concentrations corresponding to 3 mosquito equivalents and stained in the dark with 409 TMRE (5 nM) (Cell Signaling Technology) for $20 \mathrm{~min}$ at $20^{\circ} \mathrm{C}$. Images were acquired 410 without washing using an ImageStreamX Mk II (Merck Millipore) with a 60x objective 411 over $1 \mathrm{~h}$ period. To avoid a possible bias due to the variable pre-acquisition waiting 
412 times on ice, the order of sample acquisition was swapped between the experimental

413 replicates. GFP-expressing sporozoites were gated by size (Area_M01 or Area_M02)

414 and by GFP intensity (Intensity_MC_Ch02). The analysis was performed with IDEAS 6.2

415 (Merck Millipore) and FCS files were exported and analyzed by FlowJo v10. Given the

416 small size of the mitochondria, the feature finder tool from IDEAS software was used to

417 improve gating of optimally focused sporozoites. The following parameters were used to

418 discriminate in-focus single sporozoites from debris, and to identify in-focus sporozoites

419 to resolve their mitochondria: H Entropy Std_M01_Ch01_15 over H Variance

420 Std_M01_Ch01, Aspect Ratio_M02 over Symmetry 4-Object(M02_Ch2) Ch02, Gradient

421 RMS-M01-Ch01 over H Correlation Mean-M02-Ch02_3, and Gradient RMS-M02_Ch02.

422 Briefly, these parameters measure brightfield image texture, GFP image shape,

423 brightfield and GFP focus, respectively. For quantification of mitochondrial membrane

424 potential and GFP intensity, each sample was time-gated to exclude artefacts of

425 prolonged staining. The parameter corresponding to the lowest background signal in

426 unstained sporozoites was Bright Detail Intensity R7_M04_Ch04, Geo Mean which was

427 selected as bona fide TMRE intensity readout (see Figure S5D). For GFP intensity, the

428 Intensity_MC_Ch02, Geo. Mean was used. To validate the use of TMRE as a marker for

429 sporozoite membrane potential, we applied $50 \mu \mathrm{M}$ of a mitochondrial uncoupling drug

430 CCCP (Cell Signaling Technology) and showed that drug treatment abolished TMRE

431 signal in sporozoites (see Figures S4D).

\section{Statistical analysis}

434 Statistical analysis was performed with GraphPad Prism 7 software and $p$ values below 4350.05 were considered significant $\left({ }^{*}: p<0.05 ;{ }^{* *}: p<0.001 ;{ }^{* * *}: p<0.0001\right)$ and indicated in 436 the figures. The specific tests used are indicated for each figure in the corresponding 437 legend. 


\section{REFERENCES AND NOTES:}

$4391 \quad$ WHO. World Malaria Report 2016. (Licence: CC BY-NC-SA 3.0 IGO., 2016).

4402 Mideo, N., Day, T. \& Read, A. F. Modelling malaria pathogenesis. Cell Microbiol $441 \quad$ 10, 1947-1955, doi:10.1111/j.1462-5822.2008.01208.x (2008).

4423 Mideo, N. Parasite adaptations to within-host competition. Trends Parasitol 25, 443 261-268, doi:10.1016/j.pt.2009.03.001 (2009).

4444 Mideo, N. et al. Causes of variation in malaria infection dynamics: insights from 445 theory and data. Am Nat 178, E174-188, doi:10.1086/662670 (2011).

4465 Mackinnon, M. J. \& Read, A. F. Genetic Relationships between Parasite 447 Virulence and Transmission in the Rodent Malaria Plasmodium Chabaudi. Evolution 53, 689-703, doi:10.1111/j.1558-5646.1999.tb05364.x (1999).

4496 Sato, Y., Montagna, G. N. \& Matuschewski, K. Plasmodium berghei sporozoites 450 acquire virulence and immunogenicity during mosquito hemocoel transit. Infect 451 Immun 82, 1164-1172, doi:10.1128/IAI.00758-13 (2014).

4527 Attardo, G. M., Hansen, I. A. \& Raikhel, A. S. Nutritional regulation of 453 vitellogenesis in mosquitoes: implications for anautogeny. Insect Biochem Mol $454 \quad$ Biol 35, 661-675 (2005).

4558 Maier, W. A., Becker-Feldman, H. \& Seitz, H. M. Pathology of malaria-infected 456 mosquitoes. Parasitol Today 3, 216-218 (1987).

4579 Hogg, J. C. \& Hurd, H. Malaria-induced reduction of fecundity during the first gonotrophic cycle of Anopheles stephensi mosquitoes. Med Vet Entomol 9, 176180 (1995).

46010 Ferguson, H. M. \& Read, A. F. Why is the effect of malaria parasites on mosquito 461 survival still unresolved? Trends Parasitol 18, 256-261 (2002).

46211 Ferguson, H. M., Mackinnon, M. J., Chan, B. H. \& Read, A. F. Mosquito 463 mortality and the evolution of malaria virulence. Evolution 57, 2792-2804 (2003).

46412 Vezilier, J., Nicot, A., Gandon, S. \& Rivero, A. Plasmodium infection decreases 465 fecundity and increases survival of mosquitoes. Proc Biol Sci 279, 4033-4041, doi:10.1098/rspb.2012.1394 (2012). 
$46713 \quad$ Vezilier, J., Nicot, A., Gandon, S. \& Rivero, A. Plasmodium infection brings

468 forward mosquito oviposition. Biol Lett 11, doi:10.1098/rsbl.2014.0840 (2015).

46914 Bomze, I. Lotka-Volterra equation and replicator dynamics: a two-dimensional $470 \quad$ classification. Biological Cybernetics 48, 201-211 (1983).

47115 Bomze, I. Lotka-Volterra equation and replicator dynamics: new issues in $472 \quad$ classification. . Biological Cybernetics 72, 447-453 (1995).

47316 Alizon, S. \& van Baalen, M. Transmission-virulence trade-offs in vector-borne diseases. Theor Popul Biol 74, 6-15, doi:10.1016/j.tpb.2008.04.003 (2008).

47517 Mack, S. R. \& Vanderberg, J. P. Plasmodium berghei: energy metabolism of 476 sporozoites. Exp Parasitol 46, 317-322 (1978).

47718 Ponnudurai, T. et al. Sporozoite load of mosquitoes infected with Plasmodium 478 falciparum. Trans R Soc Trop Med Hyg 83, 67-70 (1989).

47919 Pollitt, L. C. et al. Costs of crowding for the transmission of malaria parasites. $480 \quad$ Evol Appl 6, 617-629, doi:10.1111/eva.12048 (2013). Neto, M. A. The major insect lipoprotein is a lipid source to mosquito stages of malaria parasite. Acta Trop 109, 159-162, doi:S0001-706X(08)002726[pii]10.1016/j.actatropica.2008.10.004 (2009). E. The major yolk protein vitellogenin interferes with the anti-Plasmodium response in the malaria mosquito Anopheles gambiae. PLoS Biol 8, e1000434, doi:10.1371/journal.pbio.1000434 (2010).

22 Ramakrishnan, C. et al. Salivary gland-specific P. berghei reporter lines enable rapid evaluation of tissue-specific sporozoite loads in mosquitoes. PLoS One 7, e36376, doi:10.1371/journal.pone.0036376 (2012). 
49825 Eickel, N. et al. Features of autophagic cell death in Plasmodium liver-stage parasites. Autophagy 9, 568-580, doi:10.4161/auto.23689 (2013).

50026 Nguyen, T. B. et al. DGAT1-Dependent Lipid Droplet Biogenesis Protects $501 \quad$ Mitochondrial Function during Starvation-Induced Autophagy. Dev Cell 42, 9-21 $502 \quad$ e25, doi:10.1016/j.devcel.2017.06.003 (2017).

50327 Boysen, K. E. \& Matuschewski, K. Arrested oocyst maturation in Plasmodium 504 parasites lacking type II NADH:ubiquinone dehydrogenase. J Biol Chem 286, 505 32661-32671, doi:10.1074/jbc.M111.269399 (2011).

506

507

508

509

510

511

$512 \quad 30$

513

514

28 Hino, A. et al. Critical roles of the mitochondrial complex II in oocyst formation of rodent malaria parasite Plasmodium berghei. J Biochem 152, 259-268, doi:10.1093/jb/mvs058 (2012).

29 Oppenheim, R. D. et al. BCKDH: the missing link in apicomplexan mitochondrial metabolism is required for full virulence of Toxoplasma gondii and Plasmodium berghei. PLoS Pathog 10, e1004263, doi:10.1371/journal.ppat.1004263 (2014).

30 Goodman, C. D. et al. Parasites resistant to the antimalarial atovaquone fail to transmit by mosquitoes. Science 352, 349-353, doi:10.1126/science.aad9279 (2016).

1 Davies, C. S., Pudney, M., Nicholas, J. C. \& Sinden, R. E. The novel hydroxynaphthoquinone 566C80 inhibits the development of liver stages of Plasmodium berghei cultured in vitro. Parasitology 106 ( Pt 1), 1-6 (1993).

32 Fowler, R. E., Sinden, R. E. \& Pudney, M. Inhibitory activity of the anti-malarial atovaquone (566C80) against ookinetes, oocysts, and sporozoites of Plasmodium berghei. J Parasitol 81, 452-458 (1995).

3 Azevedo, R. et al. A bioluminescence method for in vitro screening of Plasmodium transmission-blocking compounds. Antimicrob Agents Chemother, doi:10.1128/AAC.02699-16 (2017).

34 Goodman, C. D., Buchanan, H. D. \& McFadden, G. I. Is the Mitochondrion a Good Malaria Drug Target? Trends Parasitol 33, 185-193, doi:10.1016/j.pt.2016.10.002 (2017).

5 Gillies, M. T. The recognition of age-groups within populations of Anopheles gambiae by the pre-gravid rate and the sporozoite rate. Ann Trop Med Parasitol 48, 58-74 (1954). 
53036 Takken, W. et al. Larval nutrition differentially affects adult fitness and $531 \quad$ Plasmodium development in the malaria vectors Anopheles gambiae and 532 Anopheles stephensi. Parasit Vectors 6, 345, doi:10.1186/1756-3305-6-345 533 (2013).

53437 Moller-Jacobs, L. L., Murdock, C. C. \& Thomas, M. B. Capacity of mosquitoes to 535 transmit malaria depends on larval environment. Parasit Vectors 7, 593, 536 doi:10.1186/s13071-014-0593-4 (2014).

53738 Shapiro, L. L., Murdock, C. C., Jacobs, G. R., Thomas, R. J. \& Thomas, M. B. 538 Larval food quantity affects the capacity of adult mosquitoes to transmit human 539 malaria. Proc Biol Sci 283, doi:10.1098/rspb.2016.0298 (2016).

54039 Vantaux, A. et al. Larval nutritional stress affects vector life history traits and 541 human malaria transmission. Sci Rep 6, 36778, doi:10.1038/srep36778 (2016).

$54240 \quad$ Okech, B. A. et al. Influence of age and previous diet of Anopheles gambiae on 543 the infectivity of natural Plasmodium falciparum gametocytes from human $544 \quad$ volunteers. J Insect Sci 4, 33 (2004).

54541 O'Keefe, K. J. \& Antonovics, J. Playing by different rules: the evolution of $546 \quad$ virulence in sterilizing pathogens. Am Nat 159, 597-605, doi:10.1086/339990 547 (2002).

54842 Mitchell, S. N. \& Catteruccia, F. Anopheline Reproductive Biology: Impacts on $549 \quad$ Vectorial Capacity and Potential Avenues for Malaria Control. Cold Spring Harb $550 \quad$ Perspect Med, doi:10.1101/cshperspect.a025593 (2017).

551

43 Bourtzis, K. et al. Harnessing mosquito-Wolbachia symbiosis for vector and disease control. Acta Trop 132 Suppl, S150-163, doi:10.1016/j.actatropica.2013.11.004 (2014).

55444 Churcher, T. S. et al. Probability of Transmission of Malaria from Mosquito to 555 Human Is Regulated by Mosquito Parasite Density in Naive and Vaccinated 556 Hosts. PLoS Pathog 13, e1006108, doi:10.1371/journal.ppat.1006108 (2017).

55745 Schneider, P. et al. Submicroscopic Plasmodium falciparum gametocyte densities 558 frequently result in mosquito infection. Am J Trop Med Hyg 76, 470-474 (2007).

55946 Mendes, A. M. et al. Conserved mosquito/parasite interactions affect development $560 \quad$ of Plasmodium falciparum in Africa. PLoS Pathog 4, e1000069 (2008). 
56147 Pompon, J. \& Levashina, E. A. A New Role of the Mosquito Complement-like 562 Cascade in Male Fertility in Anopheles gambiae. PLoS Biol 13, e1002255, 563 doi:10.1371/journal.pbio.1002255 (2015).

56448 Franke-Fayard, B. et al. A Plasmodium berghei reference line that constitutively 565 expresses GFP at a high level throughout the complete life cycle. Mol Biochem 566 Parasitol 137, 23-33 (2004).

56749 Silvie, O., Goetz, K. \& Matuschewski, K. A sporozoite asparagine-rich protein 568 controls initiation of Plasmodium liver stage development. PLoS Pathog 4, 569 e1000086, doi:10.1371/journal.ppat.1000086 (2008).

57050 Lackner, P. et al. Behavioural and histopathological alterations in mice with $571 \quad$ cerebral malaria. Neuropathol Appl Neurobiol 32, 177-188, doi:10.1111/j.13652990.2006.00706.x (2006).

57351 Carroll, R. W. et al. A rapid murine coma and behavior scale for quantitative 574 assessment of murine cerebral malaria. PLoS One 5, 575 doi:10.1371/journal.pone.0013124 (2010). 


\section{ACKNOWLEDGEMENTS}

578 This work was supported by Max Planck Society, EC FP7 EVIMalaR (grant agreement $\left.579 n^{\circ} 242095\right)$ and MALVECBLOK (grant agreement $n^{\circ} 223601$ ). The authors thank $\mathrm{H}$.

580 Krüger, M. Andres and L. Spohr for mosquito rearing, mouse work assistance, and 581 Plasmodium infections, and D. Tschierske and D. Eyermann for $P$. falciparum cultures. 582 The authors express their gratitude to Prof. K. Matuschewski and his group for 583 continuous fruitful discussions, to Dr. M.M. Mota and Dr. P. Carrillo-Bustamante for 584 helpful comments.

585

\section{AUTHOR CONTRIBUTIONS}

587 GC and EAL conceived the study and designed the experiments. MG performed the 588 modeling analysis. GC, ME, RL, CG, and VB performed experiments. AEH and RS 589 contributed reagents and expertise. GC, ME, MG, RL, RS, CG, VB, and EAL analyzed 590 the data. GC, MG and EAL wrote the manuscript.

591 
$\mathbf{A}$

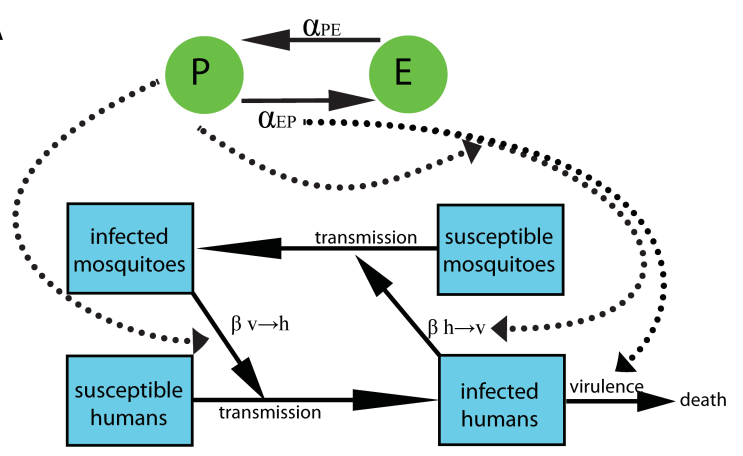

C

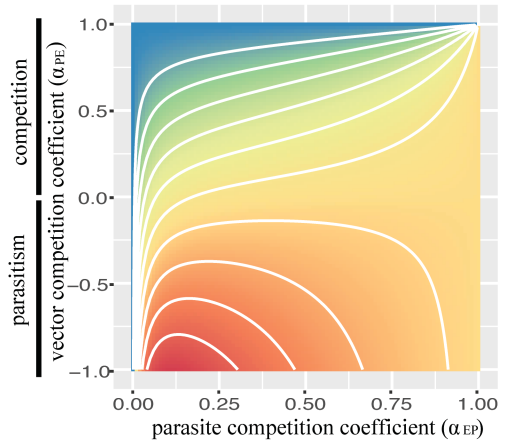

592

593

594

595

596 (A) Model scheme of parasite-vector non-mutualistic symbiotic relationship (green),

598

599

600

601

602

603

604

605

606

607

608

609
B
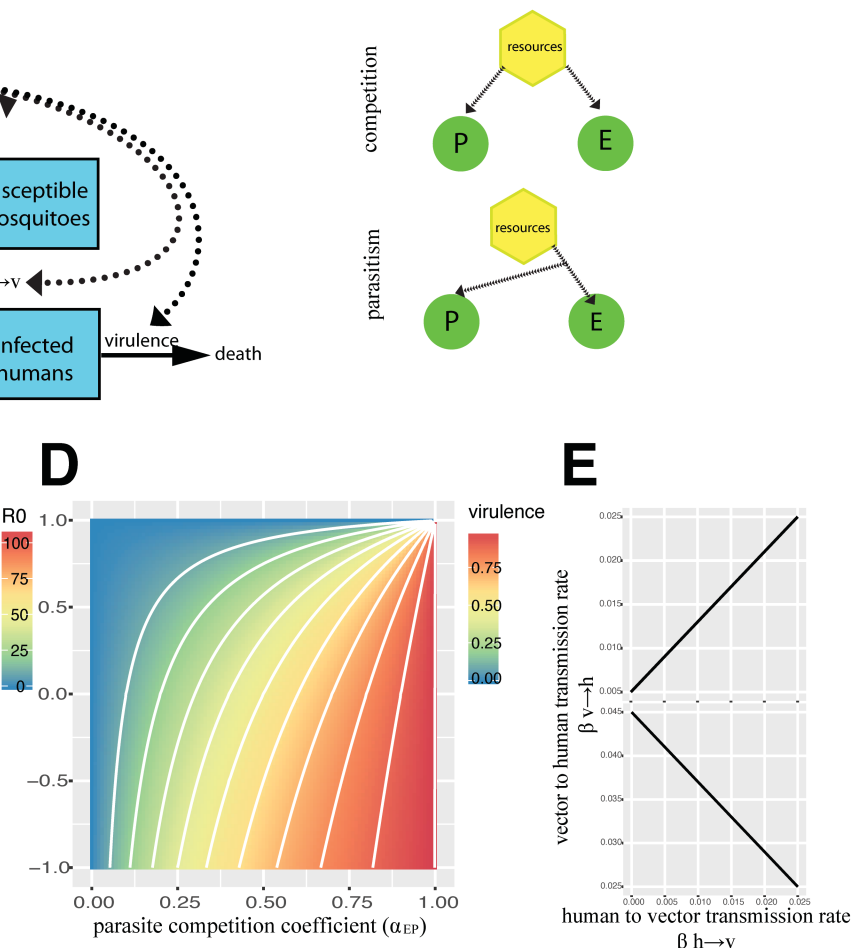

Figure 1. Evolution of vector-parasite antagonism and virulence. nested into epidemiological dynamics (blue): within-vector parasite density is positively linked to vector-to-human transmission, whereas human-to-vector transmission is a linear function of the parasite's virulence towards mosquito reproduction (competition coefficient) and human host longevity. (B) The biological relationship is competitive, if a common resource is scavenged, or parasitic if mosquito resource scavenging aids parasite development. (C) Contour plot of parasite fitness $R_{0}$ under varying within-vector interaction terms. The $y$-axis shows the relationship of mosquito egg production towards the parasite $\alpha_{P E}$ over the parasite's competition coefficient (i.e. virulence) $\alpha_{E P}$. Positive values of $\alpha_{P E}$ showcase a competitive parasite-vector interaction, whereas negative values imply that vector reproduction benefits parasite density leading to a parasitic relationship. (D) Contour plot of parasite virulence towards the host under varying withinvector interaction terms. Under competitive scenario, parasite fitness increases with virulence, whereas under parasitic scenario, an evolutionarily stable virulence strategy 
bioRxiv preprint doi: https://doi.org/10.1101/149443. this version posted October 27, 2017. The copyright holder for this preprint (which was not certified by peer review) is the author/funder, who has granted bioRxiv a license to display the preprint in perpetuity. It is made available under aCC-BY-NC-ND 4.0 International license.

610 (ESV) emerges. (E) Vector-to-human transmission over human-to-vector transmission 611 under varying degrees of parasite virulence. When mosquito and parasites 612 independently scavenge and directly compete for resources (top, $\alpha_{P E}=0.8$ ), the 613 transmission terms are positively linked. If parasites prey on mosquito resource 614 scavenging (bottom, $\alpha_{P E}=-0.8$ ), a trade-off between the transmission terms explains 615 the emergence of the ESV. P = parasites; $E=$ mosquito eggs. 

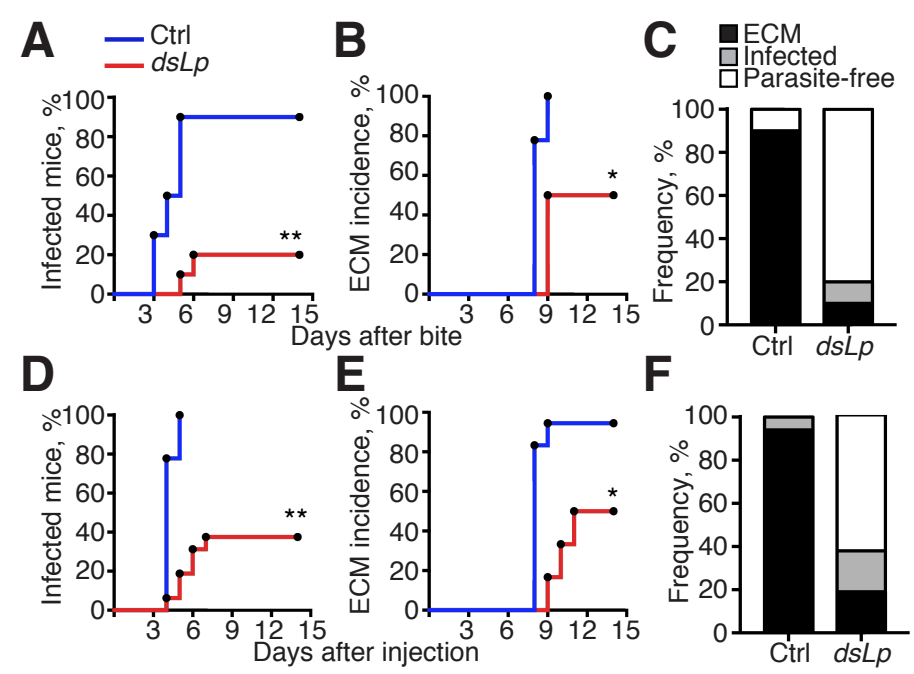

617

618 Figure 2. Within-vector environment impacts within-host Plasmodium virulence.

619

620 C57BL/6 mice infected by bites of $P$. berghei-infected mosquitoes (A-C) or by sporozoite

621 injection (D-F) were daily monitored for parasite occurrence in the blood and for

622 symptoms of experimental cerebral malaria (ECM). (A) Kaplan-Meier analysis of time to

623 infection (blood stage parasitaemia), (B) incidence (\%) of experimental cerebral malaria

$624(\mathrm{ECM})$ and $(\mathrm{C})$ cumulative health status of mice infected by bites of control $(\mathrm{Ctrl}, \mathrm{n}=10)$

625 or Lp-depleted ( $d s L p, \mathrm{n}=10$ ) mosquitoes. (D) Kaplan-Meier analysis of time to infection

626 (blood stage parasitaemia), (E) incidence (\%) of experimental cerebral malaria (ECM)

627 and (F) cumulative health status of mice infected by subcutaneous injection of 5,000

628 sporozoites dissected from the salivary glands of control (Ctrl, $n=18)$ or Lp-depleted

$629(d s L p, \mathrm{n}=16)$ mosquitoes. Asterisks indicate statistically significant differences (*: $\mathrm{p}<0.05$;

$630 * *$ : $p<0.001$; log-rank Mantel-Cox test and 2-way ANOVA). 
A

A Nile Red
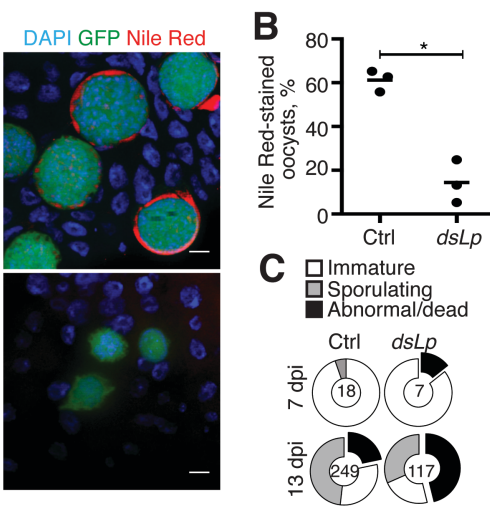

D
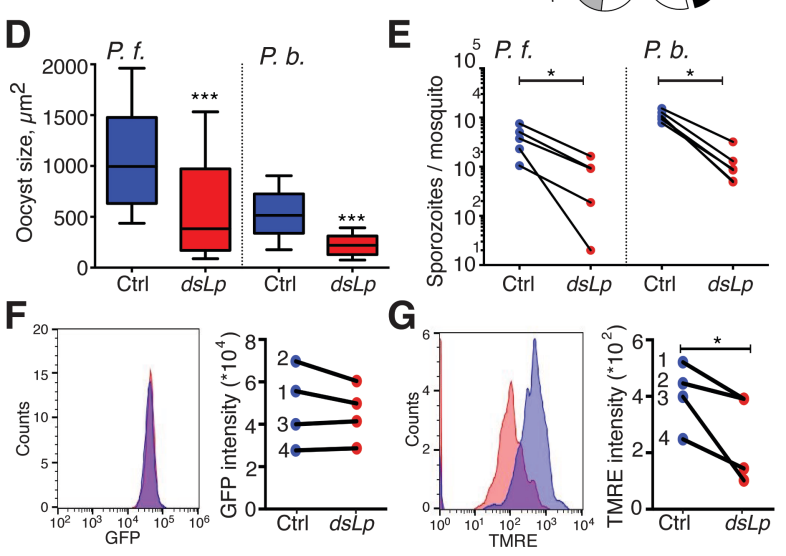

632

633 Figure 3. Mosquito lipids control within-vector oocyst proliferation, sporozoite metabolic activity and infectivity.

636 Control and Lp-deficient female mosquitoes were infected with P. berghei (P.b.) (A-G) or

637 P. falciparum (P.f.) (D, E) parasites. (A) Neutral lipids detected by the Nile Red stain in 638 the GFP-expressing P. berghei oocysts (green) $14 \mathrm{dpi}$ in control (Ctrl) and Lp-depleted 639 (dsLp) mosquitoes. Nuclei are visualized by DAPI (blue). Scale bars - $10 \mu \mathrm{m}$. (B) 640 Percentage of Nile Red-positive $P$. berghei oocysts in control and Lp-depleted 641 mosquitoes $13 \mathrm{dpi}$. At least 140 oocysts from 6 mosquitoes were analysed per condition.

642 Each point represents mean of one experiment $\left(n=3,{ }^{*}: p<0.05\right.$; paired $t$ test). (C)

643 Oocysts detected by transmission electron microscopy (see also Figure S3) were scored 644 according to the developmental stage: non-sporulating oocysts with normal morphology 645 (immature, white); oocysts with normal plasma membrane retraction, budding 646 sporoblasts, or formed sporozoites (sporulating, gray); oocysts with aberrant electron647 light intracellular vacuolization (abnormal/dead, black). Proportions of the categories are 648 shown as pie charts and the total number of analyzed oocysts per condition is indicated 649 in the middle of each chart. (D) Differences in oocyst sizes of P.f. $(11 \mathrm{dpi}, \mathrm{n}=3)$ and P.b. 
650 (14 dpi, $\mathrm{n}=6)$ in control and Lp-depleted mosquitoes. At least 225 oocysts were 651 measured per condition. The box plots represent medians (horizontal bars) with 10th 652 and 90th percentiles. (***: $p<0.0001$, Mann-Whitney test). (E) Development of P.f. (14 $653 \mathrm{dpi}, \mathrm{n}=5)$ and P.b. (18 dpi, $\mathrm{n}=4)$ salivary gland sporozoites in control and Lp-depleted 654 mosquitoes. Each point represents the mean number of sporozoites per mosquito for 655 each experimental replicate. Asterisks indicate statistically significant differences (*: $656 \mathrm{p}<0.05 ;$ 2-way ANOVA). Sporozoite (F) GFP expression and (G) mitochondrial 657 membrane potential as measured by TMRE were analysed using imaging flow 658 cytometry. Histograms from one representative experiment (left panels) and geometrical 659 mean values from four independent experiments (right panels) in control (blue, $n=1,405$ ) 660 and lipid-deprived (red, $n=593$ ) sporozoites are shown. Asterisks indicate statistically 661 significant differences ( $*$ p $<0.05$; paired $t$ test). 


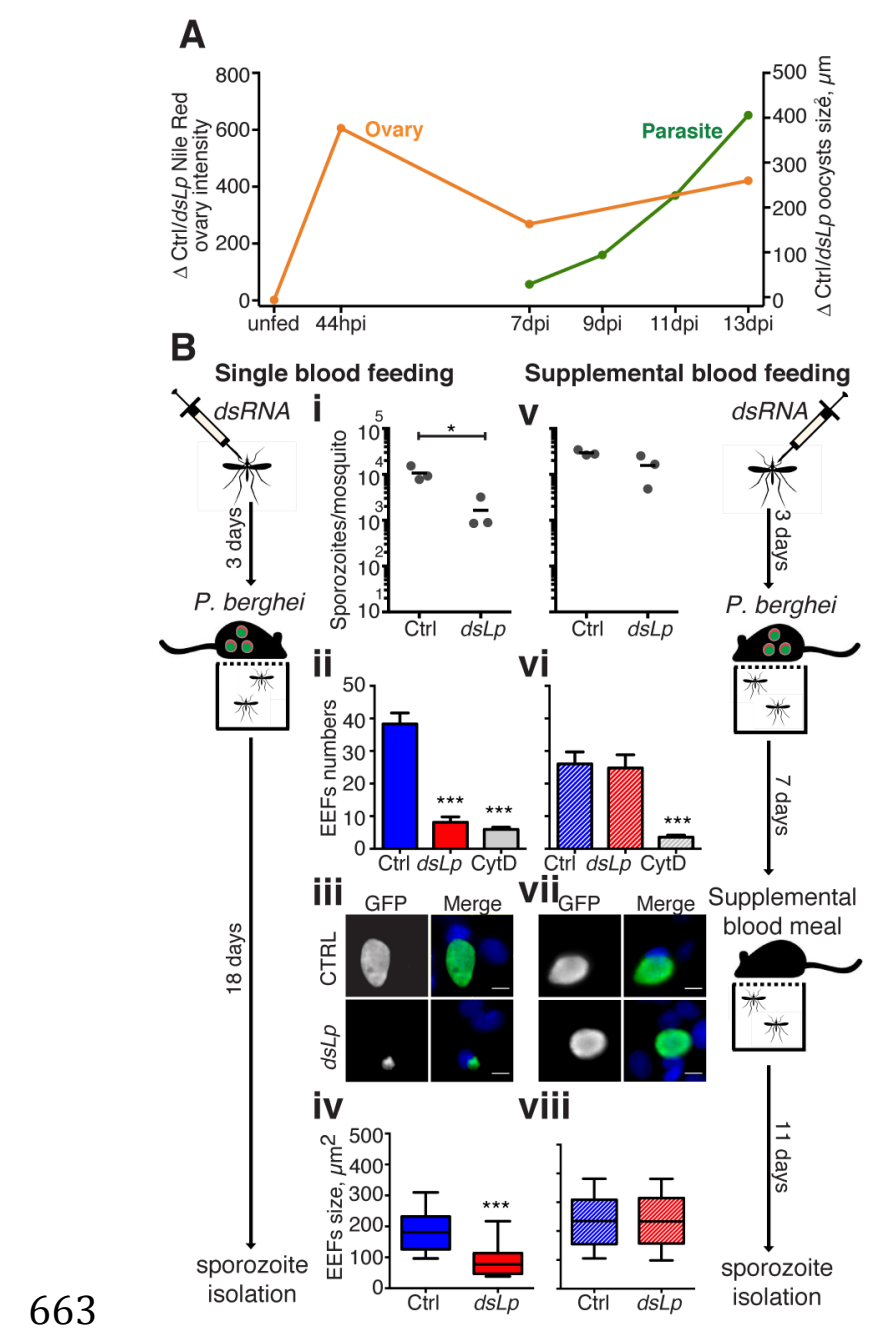

665 Figure 4. Plasmodium time-shift in sporulation circumvents competition for 666 mosquito resources.

667 (A) Kinetics of lipid acquisition by mosquito ovaries and $P$. berghei oocyst development. 668 The ratios of Nile Red mean intensities in the ovaries (orange line) and oocysts growth 669 (green line) between control and Lp-depleted mosquitoes are shown. Five or more 670 mosquitoes were dissected per time point per condition $(n \geq 2)$. (B) Lp-depleted 671 mosquitoes ( $d s L p)$ and controls (Ctrl) were infected with $P$. berghei and offered ( $\mathbf{v}$-viii) or 672 not (i-iv) a supplemental blood meal 7 days post infection. Salivary gland sporozoites 673 were isolated and counted from control and Lp-deficient mosquitoes (i and $\mathbf{v}$ ). Human 674 hepatoma HepG2 cells were infected with 10,000 sporozoites and the number of extra675 erythrocytic forms (EEFs) per field was gauged by microscopy 2 dpi (ii and vi). As a 
676 negative control, sporozoites from control mosquitoes were treated with cytochalasin D 677 (Cyt D). Mean numbers and SEM are shown. Representative fluorescent micrographs of 678 GFP-expressing EEFs (green) developing in HepG2 cells (iii and vii). Cell nuclei are 679 stained with DAPI (blue). Scale bar - $10 \mu \mathrm{m}$. EEF sizes produced by sporozoites from 680 control and Lp-deficient mosquitoes (iv and viii). The box plots represent 10th and 90th 681 percentiles while the horizontal lines show medians. Asterisks indicate statistically 682 significant differences $\left(n=3,{ }^{*}: p<0.05\right.$; ${ }^{* * *}: p<0.0001$; Kruskal-Wallis and Mann-Whitney 683 test). 